\title{
Untersuchungen über Zusammensetzung und Transport von Schwebstoffen n einigen Schweizer Flüssen
}

\author{
Barbara E. Peters-Kümmerly
}

\section{Abstract}

The amount and composition of suspended load from 19 stations in Swiss rivers were studied. Generally, the correlation between the amount of suspended load and of water discharge is good, although measurements over short periods of time show large fluctuations.

The denudation rate was calculated from the suspended load discharge, the amount of elements in solution, and the water discharge. Values of about $0.05 \mathrm{~mm} / \mathrm{yr}$ were obtained for drainage areas consisting mainly of crystalline rocks and about $0.25 \mathrm{~mm} / \mathrm{yr}$ for drainage areas dominated by sedimentary rocks.

The mineralogical composition of the suspended load determined by $X$-ray diffraction can be correlated with the rock types in the drainage areas. Composition changes resulting from transportation were not observed. The clay fraction is mainly composed of illite and chlorite which was, for the most part, formed during mechanical weathering in the Alps.

\section{Problemstellung}

Im Anschluß an limnologische Untersuchungen an verschiedenen Schweizer Seen und hydrologische Untersuchungen des Geographischen Institutes Bern sowie sedimentologische und tonmineralogische Untersuchungen an Glazialablagerungen des Geologischen und Mineralogisch-Petrographischen Institutes drängte sich die Untersuchung der Zusammensetzung der Schwebstoffe rezenter Flüsse auf. Folgende Fragen standen dabei im Vordergrund:

- Aus welchen kristallisierten Phasen sind die Schwebstoffe aufgebaut, und wie lassen sich die qualitativen Unterschiede mit den Einzugsgebieten korrelieren?

- Lassen sich innerhalb einer Entnahmestelle Unterschiede in der Schwebstoffzusammensetzung beobachten?

- Wie beziffert sich die Abtragung in den verschiedenen Einzugsgebieten, und wie lassen sich die ermittelten Beträge mit dem jeweiligen Gesteinsaufbau korrelieren?

\section{Geographische Übersicht}

Das Eidgenössische Amt für Wasserwirtschaft begann im Juli des Jahres 1962 mit der Entnahme von Schwebstoffproben an folgenden Stellen: Aare Brugg, Aare Brügg, Aare Stilli, Inn Martinsbruck und Thur Bütschwil. Das Stationennetz wurde dann ausgebaut und umfaßt heute bereits 19 Entnahmestellen (Tab. 1).

Auf Tabelle 1 sind die Prozentsätze der Vergletscherung in den einzelnen Gebieten zu finden. Die höchstgelegenen Gebiete, Lonza Blatten und Borgne La Luette, sind zu 40 und $25 \%$ vergletschert und vereist.

Das Klima der Schweiz wird geprägt durch die Einwirkungen von ozeanischen, nordeuropäischen, kontinentalen und mittelmeerischen Klimaeinflüssen. Die Gebirgsketten verunmöglichen eine homogene Mischung dieser Typen und bewirken eine Aufteilung in begrenzte Klimaregionen. Zwischen diesen können größere Klimaunterschiede auftreten.

Trotz der erwähnten Klimaunterschiede kann ganz allgemein gesagt werden, daß die mittlere monatliche Schwebstoffbelastung über mehrere Jahre, in den meisten Flüssen im Frühsommer infolge von Hochwasser und Schneeschmelze am höchsten und in den Wintermonaten am niedrigsten ist (Fig. 8). Dies bestätigen Whetten, Kelley und Hanson (1969) in ihrer Arbeit über Untersuchungen am Columbia River. Ihr Einzugsgebiet unterliegt den Einflüssen des ozeanischen Klimas. In den Jahren mit durchschnittlicher Abflußmenge (ohne Extremwerte) und demzufolge durchschnittlicher Schwebstoffkonzentration finden sie das Konzentrationsmaximum an Schwebstoffen in den Sommermonaten. Die drei Autoren weisen aber auch auf Jahre mit Extremwerten hin, wo in einigen Tagen oder Wochen, als Folge von Hochwasser, der größte Teil des Jahresfeinstoffmaterials transportiert wird. Nach Rantz und Moore (1965) betrugen die maximalen Schwebstoffkonzentrationen während der Überschwemmungen im unteren Columbia-Lauf im Dezember des Jahres 1964 zehnmal mehr als die Höchstkonzentration eines durchschnittlichen Jahres. 
Tabelle 1: Flächenausdehnung und Vergletscherung der Einzugsgebiete Höhenlage der Einzugsgebiete und der Entnahmestellen

\begin{tabular}{|c|c|c|c|c|c|c|}
\hline Gewässer & Entnahmestelle & Abkürzung & 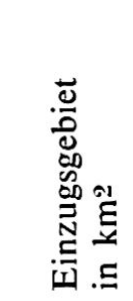 & 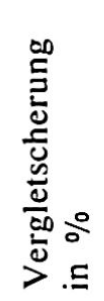 & 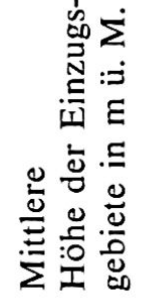 & 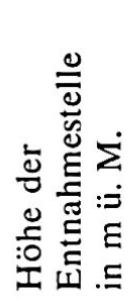 \\
\hline Rhone & Brigerbad & RB (VS) & 913 & 27,1 & 2370 & 667 \\
\hline Linth & Weesen Gäsi & LWG & 616 & 5,3 & 1720 & 423 \\
\hline Inn & Martinsbruck & IM & 1945 & 5,4 & 2350 & 1030 \\
\hline Lütschine & Bönigen (Gsteig) & LB & 379 & 19,5 & 2050 & 585 \\
\hline Lonza & Blatten & LB (VS) & 78 & 40,6 & 2630 & 1520 \\
\hline Aare & Brienzwiler & B & 554 & 24,4 & 2150 & 570 \\
\hline Rhein & Bad Ragaz & RhBdR & 4455 & 2,5 & 1930 & 497 \\
\hline Thur & Bütschwil & TB (SG) & 303 & 0,0 & 1110 & 565 \\
\hline Arve & Genève & $A(G E)$ & 1983 & 6,1 & 1400 & 372 \\
\hline Rhein & Schmitter & $\mathrm{RhSm}$ (SG) & 6119 & 2,0 & 1800 & 407 \\
\hline Grande Eau & Aigle & GA (VD) & 132 & 1,9 & 1560 & 414 \\
\hline Borgne & La Luette & $\mathrm{BL}$ (VS) & 231 & 25,0 & 2620 & 957 \\
\hline Aare & $\begin{array}{l}\text { Interlaken } \\
\text { Unterseen }\end{array}$ & $\mathrm{U} \quad:$ & 1140 & 18,2 & 1950 & 559 \\
\hline Linth & Weesen Biäsche & LWB & 1061 & 3,1 & 1580 & 419 \\
\hline Aare & Stilli & AS (AG) & 17625 & 2,4 & 1050 & 326 \\
\hline Rhône & Porte du Scex & RPS & 5220 & 16,2 & 2130 & 377 \\
\hline Rhône & Genève & $\mathrm{R}(\mathrm{GE})$ & 7987 & 10,6 & 1670 & 369 \\
\hline Aare & Brugg & $\mathrm{AB}(\mathrm{AG})$ & 11750 & 2,4 & 1010 & 332 \\
\hline Aare & Brügg & $\mathrm{AB}(\mathrm{BE})$ & 8317 & 3,3 & 1150 & 428 \\
\hline
\end{tabular}

\section{Probenahme}

An den 19 Entnahmestellen werden Schwebstoffsowie Wassertemperatur- und Abflußmengen mit Pegel, Limnigraph und speziellen Wasserentnahmegeräten, durchschnittlich sieben- bis zehnmal pro Monat, vorgenommen. Uns interessierten ausschließlich die Ergebnisse der Schwebstoffmessungen.

Das hier verarbeitete Untersuchungsmaterial wurde als Schwebstoffwassermischung mittels eines Schwebstoffprobe-Entnahmegerätes in der Mitte des Flusses, ungefähr $1 \mathrm{~m}$ unter dem Wasserspiegel entnommen. Das Gerät ist im Eidgenössischen Amt für Wasserwirtschaft entwickelt worden.

Es gibt zwei Grundmethoden zur Bestimmung der Schwebstoffkonzentration in einem Vertikalprofil des Flusses: die tiefenintegrierte Probenahme und die punktintegrierte Probenahme. In unserem Falle wurde meistens nach der letzteren gearbeitet. Während bei der Tiefenintegration die Entnahme-Apparatur kontinuierlich, mit annähernd gleichmäßiger Geschwindigkeit von der Wasseroberfläche bis an einen Punkt nahe dem Flußbett gesenkt wird und so in jedem Punkt, proportional der Strömungsgeschwindigkeit, die Wasserfeststoffmischung in das Sammelgefäß eintritt, findet dieser Vorgang bei der Punktintegration an einem festen Punkt statt. Im

allgemeinen erreicht man bei der Punkt-Probeentnahme mit der Zunahme der Anzahl Proben eine größere Genauigkeit. Es gibt aber mehrere Faktoren, die berücksichtigt werden müssen, um einen möglichst kleinen Fehlerprozentsatz anzustreben. Nach Untersuchungen am Missouri River (Eidg. Amt für Wasserwirtschaft, Reports Nr. 7 [1943] und 14 [1963]), kann die Schwebstoffkonzentration in wenigen Sekunden stark variieren und deshalb sind Sammelapparaturen, die über längere Zeit Wasser aufnehmen können, erforderlich. Die Entnahmen des Wasserwirtschaftsamtes dauern erfahrungsgemäß 30 Minuten. Andere Faktoren, welche die Untersuchungsergebnisse beeinflussen können, sind die zunehmende Partikelgröße, mehr Schwebe in größeren Tiefen, geringe Geschwindigkeit des Flusses und Veränderungen der Turbulenz. Es sind demnach mehrere Bedingungen zu erfüllen, um repräsentative Proben zu erhalten. Der Fehler der im Moment in der Schweiz verwendeten Methode dürfte nicht mehr als $50 \%$ betragen.

Nachdem die Wasserfeststoffproben gesammelt sind, werden sie in Plastikflaschen ins Labor gesandt. Dort läßt man die Proben auf Membranfiltern abfiltrieren, schreibt sie an und wägt sie ein. Einige Milligramm trockenes Feststoffmaterial befinden sich auf den kleinen Filtern. Dieses Material steht für Untersuchungen zur Verfügung. 
Die Abflußmenge pro Zeiteinheit $\left(\mathrm{m}^{3} / \mathrm{s}\right)$ wird aus den Teilflächen des Meßquerschnittes $\left(\mathrm{m}^{2}\right)$ und den Strömungsgeschwindigkeiten $(\mathrm{m} / \mathrm{s})$ ermittelt. Auch hier wird meist ein Punktmeßverfahren gebraucht, wobei an mehreren Punkten des Meßquerschnittes Geschwindigkeitsmessungen vorgenommen werden. Heute erfolgt die Auswertung dieser Flügelmessungen nicht mehr nach dem graphischplanimetrischen Verfahren, sondern auf elektronischen Weg (Eidg. Amt für Wasserwirtschaft 1967).

\section{Untersuchungsmethodik}

Wegen der Feinheit der Proben war man für die Mineralbestimmung auf das Röntgen angewiesen. Für andere Verfahren wie DTA (Differential-Thermoanalyse), TG (Thermogravimetrie) usw. stand zu wenig Material zur Verfügung. Im allgemeinen haben wir zwei Röntgenmethoden verwendet: Mit der Guinier- und der Diffraktometer-Methode sind die 19 Entnahmestellen auf ihre Mineralzusammensetzung hin bearbeitet worden.

\section{Mineralbestand der Gesamtproben:}

Mit der Guinierkamera wurden von 19 gemörserten Schwebstoffproben nach de Wolff (1951) Aufnahmen gemacht, auf welchen, je nach den verschiedenen Intensitäten der Mineralien, verschieden starke Reflexe photographisch registriert sind. Nebst diesen qualitativen Bestimmungen ergaben sich hier erste Anhaltspunkte für quantitative Anteile der Mineralien. Anschließend fand die Untersuchung der Lithiumfluorid-Gesamtproben-Mischung auf dem Diffraktometer statt. Das Diffraktometer registriert die Intensitäten der Reflexe elektronisch und zeichnet diese für die verschiedenen Mineralien mittels eines Schreibers direkt auf (Fig. 1). Nach der Methode von Klug und Alexander (1954) lassen sich die Gewichtsprozent-Anteile ermitteln.

Beim Vergleich von Resultaten der beiden Röntgenverfahren stimmten die Mineralgehalte weitgehend überein.

\section{Mineralbestand der Tonfraktion:}

Grundlegend anders war hier die Vorbereitung der Präparate. Man ließ die wieder von den Filtern in Suspension gebrachten Proben während 2 Stunden absedimentieren. Dann wurde $2 \mathrm{~cm}$ unter der Lösungsoberfläche die Fraktion $<2 \mu$ abpipetiert und auf Glasplättchen verdunstet. Diese Glasplättchen, mit dem z. T. äußerst spärlichen Material darauf, dienten unserer weiteren Untersuchung.

Sie wurden nun auf dem Diffraktometer geröntgt.

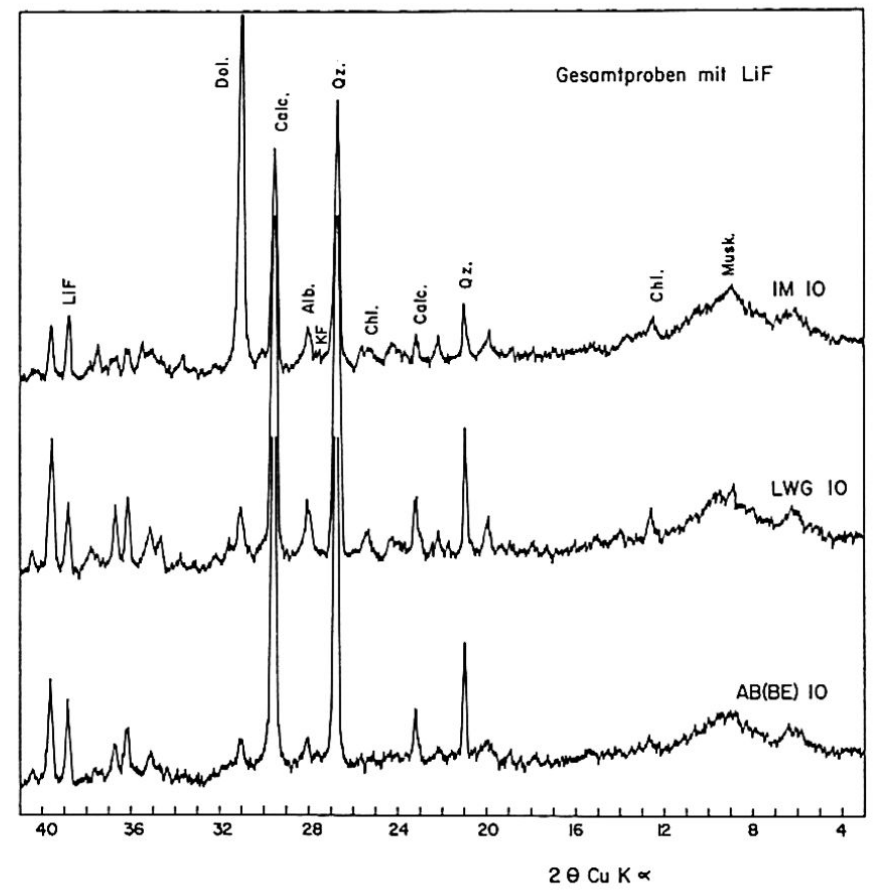

Figur 1. Diffraktometerdiagramme einiger $\mathrm{Ge}$ samtproben. Unorientierte Präparate

Abkürzungen: LiF Lithiumfluorid, Dol. Dolomit, Calc.Calcit, Alb.Albit, KF Kalifeldspat, Qz.Quarz, Chl. Chlorit, Musk. Muskowit

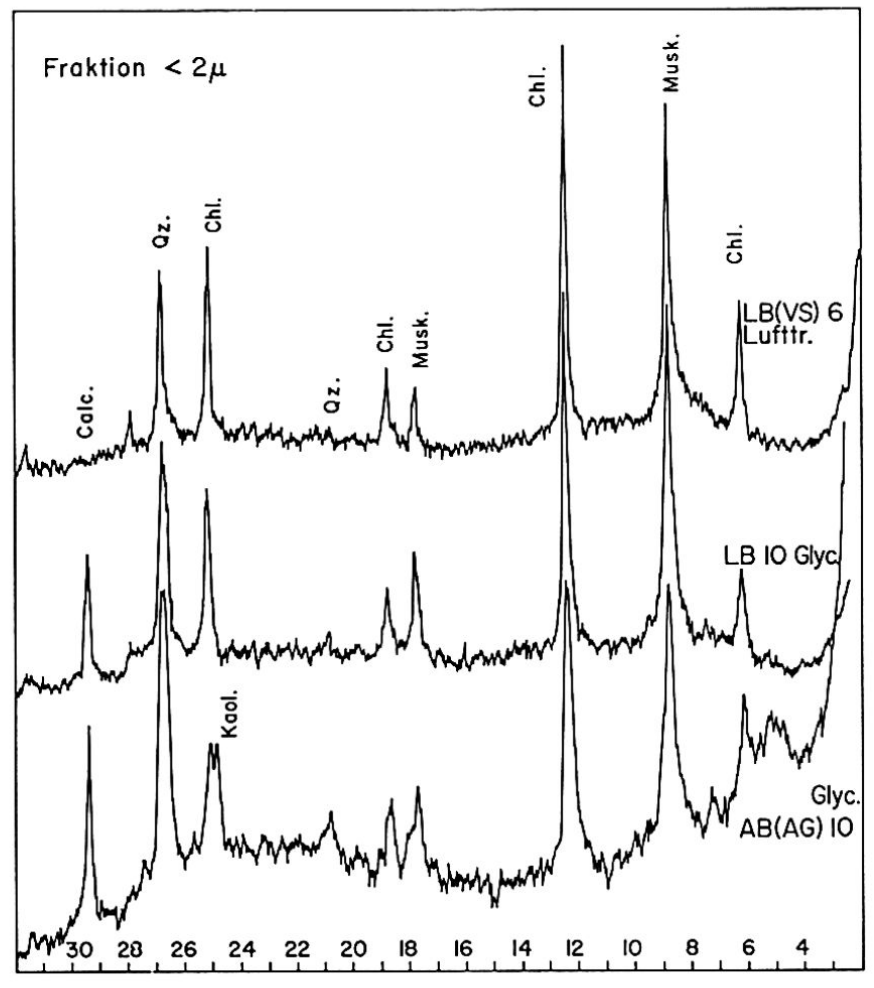

$2 \theta \mathrm{CuK} \alpha$

Figur 2. Diffraktometerdiagramme einiger Tonproben (Fraktion $<2 \mu$ ). Texturaufnahmen bei verschiedenen Aufnahmebedingungen

Abkürzungen: Calc. Calcit, Qz. Quarz, Kaol. Kaolinit, Ch. Chlorit, Musk. Muskowit, Lufttr. Lufttrocken, Glyc. mit Äthylenglycol 
Es handelte sich hier um orientierte Präparate, welche mit $\mathrm{CuK}_{\alpha}$-Strahlung geröntgt wurden, und zwar alle lufttrocken, der größte Teil mit Äthylenglycol und ein kleiner Teil gebrannt bei $550 \mathrm{C}^{\circ}$ (Fig. 2).

Das Probenmaterial der lufttrocken geröntgten
Glasplättchen wurde dann für die noch folgenden Guinieraufnahmen verwendet.

Auf diese Art konnten wir die Mineralien der Tonfraktion bestimmen und die Anteile der verschiedenen Mineralien nach der Methode von Johns, Grim und Bradley (1954) abschätzen.

\section{ALLGEMEINER TEIL}

\section{A. Gesamtproben}

Die Minerale der Schwebstoffgesamtproben, in Fig. 3 (Seite 144/145) graphisch dargestellt und in den Tabellen 2 und 3 in Gewichtsprozenten aufgeführt, sind nach den anfangs beschriebenen Methoden röntgenographisch ermittelt worden und sollen nun besprochen werden.

In der Mehrzahl der Proben überwiegt der Muskowitanteil. Es folgen Quarz, Karbonate (mit hauptsächlich Calcit und geringeren Mengen von Dolomit), Feldspate (mit vorherrschendem Albitgehalt und wenig Kalifeldspat), Chlorit und - zuletzt meistens nur in Spuren auftretend - Biotit, Amphibol und Talk oder Pyprophyllit. Úber die Tonmineralanteile, welche aus den Untersuchungen der Tonfraktion $<2 \mu$ hervorgingen, wird im nächsten Kapitel berichtet.

Der Karbonatgehalt schwankt in den Durchschnittsproben der 19 Stationen zwischen $0 \%$ in der Entnahmestelle Lonza Blatten und 44\% in der Entnahmestelle Inn Martinsbruck. Der mittlere Gehalt liegt ungefähr bei $20 \%$. Hiervon sind ungefähr $3 / 4$ Calcit und $1 / 4$ Dolomit, was nur einen allgemeinen Anhaltspunkt geben kann, weil das Calcit/ Dolomit-Verhältnis der verschiedenen Entnahmestellen ziemlich stark variiert. Es ist besonders gering in Gebieten, in denen die Triasvorkommen viel Dolomit aufweisen (Inn Martinsbruck), und hoch in Gegenden der Kreide- und Juraformationen, deren Kalke hohe Calcitgehalte haben (Linth Weesen Gäsi).

Müller und Förstner (1968) haben festgestellt, daß eine Abhängigkeit zwischen den Karbonatgehalten und den Durchflußmengen besteht, wenn letztere in Werte bei ansteigendem und abfallendem Hochwasser unterteilt werden. Es fallen bei gleichen Wasserständen in ansteigenden Hochwassern größere Karbonatmengen an als in abfallenden Hochwassern.

Quarz und die Feldspate bilden zusammen einen großen Teil des Mineralbestandes. Der Quarzanteil variiert in den Durchschnittsproben zwischen 11\% (Inn Martinsbruck) und 30\% (Lütschine Bönigen), der Albit zwischen 4\% (Thur Bütschwil) und $28,5 \%$ (Rhone Brigerbad), der Kalifeldspat fehlt in mehreren Stationen und ist mit einem maxima- len Wert von $12 \%$ in der Entnahmestelle Aare Brienzwiler vertreten. Als Richtlinie können wir ungefähr ein Verhältnis von $25 \%$ Quarz zu $15 \%$ Albit zu $<5 \%$ Kalifeldspat angeben. Der dem Kalifeldspat gegenüber recht hohe Gehalt an Albit tritt vorwiegend in Gneisen auf und ist ziemlich beständig. Er erklärt sich aus einer Neubildung bei niedriger Metamorphose in den nördlichen Alpen (Wenk 1962). Der Kalifeldspat kommt fast nur in granitischen Gesteinen der kristallinen Gebiete vor und wird, einmal der Verwitterung ausgesetzt, relativ rasch umgewandelt. Calciumreichen Plagioklas konnten wir keinen feststellen. Dieser ist in den Alpen meist stark saussuritisiert und stark verwitterungsanfällig.

Die Chloritanteile bewegen sich zwischen 5\% (Rhone Brigerbad) und 21\% (Aare Interlaken/Unterseen). Auf die Chloritgehalte und ihre mögliche Herkunft ist im Kapitel «Detailuntersuchıngen einiger Entnahmestellen», Diss. Peters (1971), eingegangen worden.

Bei den hellen Glimmern haben wir unterschieden zwischen Muskowit und einem Gemisch von feinkörnigem Muskowit mit Illit, was in der Fig. 3 deutlich zum Ausdruck kommt. Diese Mischungen treten vorwiegend im Mittelland auf. Sie dürften aus dem stark Illit führenden Molasseuntergrund aufbereitet worden sein.

\section{B. Tonmineralbestand}

Der Tonmineralbestand, welcher in der Fig. 4 dargestellt und in der Tabelle 4 aufgeführt ist, wurde ebenfalls mit den im methodischen Teil beschriebenen Verfahren röntgenographisch ermittelt. Von den Schwebstoffproben der 19 untersuchten Entnahmestellen enthalten 13 nur die Komponenten Illit und Chlorit. Die restlichen sechs Proben weisen vier verschiedene Mineralvergesellschaftungen auf. Die Minerale der ersten Assoziation (Rhône Porte du Scex) sind Illit, Chlorit und Kaolinit, jene der zweiten (Rhone Brigerbad) Illit, Chlorit und wenig Mixedlayer-Minerale (Tonminerale mit Wechsellagerungsstrukturen). Weiter findet man im Molassetrog eine dritte Gruppe (Aare Brügg und Thur 
Bütschwil) mit Illit, Chlorit, Kaolinit und Mixedlayer-Mineralien, dann ebenso in der Molasse eine vierte Vergesellschaftung (Aare Brugg und Aare Stilli) mit Illit und prozentual abnehmendem Chlorit, Kaolinit und Montmorillonit.

Bei den ersten acht Entnahmestellen in der Tabelle
4 sind die semiquantitativen Resultate der Tonmineralbestände von einzeln geröntgten Filterproben mit verschiedenen Mengen Material von unterschiedlicher Farbe sowie von je einer Durchschnittsprobe von 20 Filterproben dargestellt. Für die restlichen Stationen wurden nur Durchschnitts-

Tabelle 2: Mineralbestand der Gesamtp̈oben

Nr. 1, 2, 3 und 4 sind Einzelproben und Nr. 10 sind Durchschnittsproben von 20-60 Filtern

Mineralbestand in Gew. \%

Entnahmestelle

Rhône Brigerbad

Linth Weesen Gäsi

Inn

Martinsbruck

Lütschine Bönigen

Lonza Blatten

Aare

Rhein

Bad Ragaz

Thur

Bütschwil

Borgne

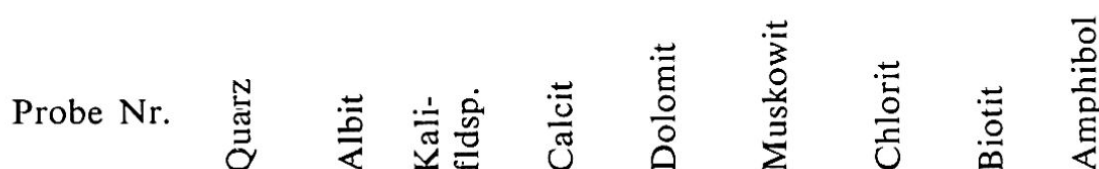

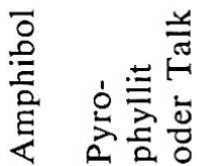

20

25-30 10

$\mathrm{sp}$

- $30 \quad 5$

$\begin{array}{llllll}25 & 10 & 5 & \text { sp } & 20-25 & 5\end{array}$

25-30 10

25-30 10

$29 \quad 10$

sp

sp

- $30 \quad 5$

sp - $23 \quad 5$

25-30 10

sp 30

30

25-30 10

30-35 10

25-30 10

sp $\quad 30$

sp 20

2010

5-10- -

5-10- -

5-10- -

5-10- -

$5-10--$ 
werte anhand der Präparate mit Material von 20 bis 60 Filterproben ermittelt.

Im allgemeinen konnten nur minime quantitative Unterschiede innerhalb einer Entnahmestelle, wie auch zwischen den Einzel- und den Durchschnittsproben verschiedener Stationen, festgestellt werden. Man dürfte demzufolge annehmen, daß bei einem Hochwasser der Tonmineralbestand unverändert bleibt. Dagegen berichten Müller und Förstner (1968), wie in Trübstoffen des Alpenrheins zu Beginn des Frühsommer-Hochwassers im Jahre 1965 plötzlich ein zusätzliches Wechsellagerungsmineral auftrat. Im übrigen stellten sie während des ganzen Beobachtungszeitraumes nur Illit und Chlorit fest. In den Proben aller Entnahmestellen herrscht Illit vor. Da die Einzugsgebiete im Kristallin der Alpen, in den mesozoischen Sedimenten und in den Sedimenten der Molasse liegen, stellt sich die Frage nach der Herkunft von Illit und Chlorit. Wird die Kristallinität von Illit nach der Methode von Kubler (1967) gemessen, so weisen bei unseren Proben nur jene von Grande Eau Aigle eine schlechte Kristallinität auf. Dies ist wohl auf die großen Flyschvorkommen im Einzugsgebiet zurückzuführen. Da hauptsächlich in den Alpen durch mechanische Verwitterung gut kristallisierte Illite und Chlorite entstehen, während die Kristallinität in den Molassesedimenten schlecht ist, dürften unsere Illit-Anteile eher aus dem Hinterland der Flüsse, den Alpen, stammen. Kaolinit stellten wir in fünf Proben fest. Bisher fanden Vernet (1959) und Peters (1969) in den Sedimenten der schweizerischen Molasse eher wenig Kaolinit. Nach Peters (1969) tritt er nur in den Sandsteinen der Bodenseeschüttung und nach Füchtbauer (1964) in den Molassesedimenten östlich des Bodensees häufig auf. Ob das Kaolinitvorkommen der Entnahmestelle Thur Bütschwil aus dem Unter- und Mittellauf des Flusses oder aus dem Hinterland stammt, bleibt hier ungeklärt. Die Kaolinitgehalte der Entnahmestellen Aare Brügg, Brugg und Stilli dürften durch Zuflüsse aus den Kaolinit führenden, mesozoischen und tertiären Sedimenten des Jura bedingt sein. In einer Probe aus den Alluvionen bei der Rhonemündung in den Genfersee konnte Vernet (1959) Kaolinit feststellen, aber nicht eindeutig nachweisen und herleiten. Das Kaolinitvorkommen der Schwebstoffentnahmestelle Rhône Porte du Scex dürfte auf die Walliser Karbonzone des Aiguilles-Rouges-Massivs zurückgeführt werden.

Berry, Brophy und Naquash (1970), die Kaolinit im Einzugsgebiet des Euphrats, nicht aber im Einzugsgebiet des Tigris feststellten, führten die Tatsache auf die klimatischen Unterschiede in den beiden Gebieten zurück.

Nach Vernet (1959) und Peters (1969) findet man sehr viel Montmorillonit in den Molassesedimenten. Nachdem die Aare und deren Zuflüsse die Molasse durchquert haben, weisen sie in den Schwebgutproben der Entnahmestellen Aare Brugg und Aare Stilli nur wenig Montmorillonit auf. Demzufolge sind es nicht die Molassesedimente, die mittransportiert werden, sondern, wie oben schon erwähnt, vor allem Illit und Chlorit aus dem Hinterland der Flüsse. Daraus kann geschlossen werden, daß ein Großteil der Tonmineralien trotz der Seen nicht abgelagert, sondern weitertransportiert wird.

\section{Schwebstoff belastung uud Abtragung \\ 1. Beziehung Schwebstoffkonzentration - Durch- flußmenge}

Hier befassen wir uns mit der Schwebstoffbelastung bzw. der Schwebstoffkonzentration und ih-

Tabelle 3: Mineralbestand der Gesamtproben von 11 weiteren Entnahmestellen (Durchschnittsproben) in Gew. \%.

Entnahmestelle

$\begin{array}{ll}\text { Arve } & \text { Genève } \\ \text { Rhein } & \text { Schmitter } \\ \text { Grande Eau } & \text { Aigle } \\ \text { Borgne } & \text { La Luette } \\ \text { Aare } & \text { Interlaken } \\ \text { Linth } & \text { Unterseen } \\ \text { Aare } & \text { Weesen Biäsche } \\ \text { Rhône } & \text { Stilli } \\ \text { Rhône } & \text { Porte du Scex } \\ \text { Aare } & \text { Genève } \\ \text { Aare } & \text { Brugg (AG) } \\ & \text { Brügg (BE) }\end{array}$

N
Ð
29
23
20
24
17
16
27
21
21
26
17

\begin{tabular}{|c|c|c|}
\hline$\frac{\mathrm{L}}{\frac{0}{2}}$ & 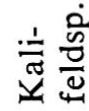 & c5 \\
\hline 13 & - & 18 \\
\hline 7 & - & 21 \\
\hline 6 & - & 21 \\
\hline 18 & - & 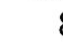 \\
\hline 10 & - & 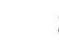 \\
\hline 8 & - & 17 \\
\hline 8 & - & 18 \\
\hline 15 & - & $\varepsilon$ \\
\hline 10 & sp & 13 \\
\hline 8 & - & 17 \\
\hline 6 & - & 2 \\
\hline
\end{tabular}

\begin{tabular}{|c|c|c|c|c|}
\hline 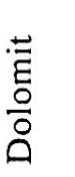 & 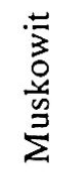 & $\frac{\vec{E}}{\frac{\vec{g}}{J}}$ & 苛 & \\
\hline 3 & 26 & 11 & $\mathrm{sp}$ & - \\
\hline 9 & 31 & 10 & - & \\
\hline 4 & 28 & 21 & - & - \\
\hline 3 & 32 & 15 & - & sl \\
\hline $1-2$ & 36 & 21 & $<5$ & \\
\hline 3 & 36 & 20 & - & $\ldots$ \\
\hline & 26 & 18 & - & \\
\hline 4 & 36 & 16 & - & - \\
\hline 3 & 37 & 16 & - & \\
\hline $2-3$ & 28 & 18 & - & \\
\hline & 37 & 15 & - & \\
\hline
\end{tabular}


rem Verhältnis zur Wasserführung der Flüsse. Wir berufen uns auf die Fig. 5, 6 und 7, welche die Punktwolken einiger Entnahmestellen zeigen. Die durch diese Punktwolken gelegten Geraden sind in der Fig. 9 dargestellt. Es gilt für sie die allgemeine Gleichung

$$
\mathrm{C}_{\mathrm{s}}=\mathrm{a} \cdot \mathrm{Q}^{\mathrm{b}}
$$

Tabelle 4

Entnahmestelle

Rhône Brigerbad

$\mathrm{C}_{\mathrm{s}}=$ Schwebstoffkonzentration, $\mathrm{Q}=$ Abfluß-

menge, $\mathrm{a}, \mathrm{b}=$ Exponenten

Im großen und ganzen prägt sich die Abhängigkeit der Schwebstoffmenge vom Wasserdurchfluß deutlich aus, wenn die Daten über längere Zeit gemessen wurden. Bei Ereignissen von kürzerer Beobachtungsdauer stellen Nydegger (1967) sowie Müller und Förstner (1968) fest, daß oft gar keine oder unterschiedliche Zusammenhänge bestehen. Nydegger konnte bei geringem Abfluß (z. B. in den Wintermonaten) keine Abhängigkeit der Schwebstoffbelastung vom Durchfluß feststellen. Jeder Fluß habe seine kritische Fließgeschwindigkeit, unterhalb welcher andere Gesetzmäßigkeiten gelten als oberhalb. Wir können die stark unterschiedlichen Schwebstoffgehalte zwischen den untersuchten Gesamtproben der Entnahmestelle Inn Martinsbruck (Tabelle 2) nicht auf sommerliche Hochwasser zurückführen, da zusätzliche Untersuchungen an Proben der Monate Oktober/November auch verschiedene Resultate ergaben. Müller und Förstner (1968) zeigen anhand von Diagrammen von drei aufeinanderfolgenden Hochwassern, wie beim ersten die höchste Schwebstoffkonzentration 8 bis 12 Stunden vor dem Maximum des Wasserdurchflusses auftritt, während schon beim folgenden zweiten Hochwasser die Kurven der Schwebstoffkonzentration und des Wasserdurchflusses nahezu parallel verlaufen. Die beiden Autoren erklären die beim ersten Hochwasser unterschiedliche Abhängigkeit der Schwebstoffmenge vom Durchfluß durch eine gründliche Ausschwemmung jeglichen Lockermaterials, welches in Zeiten mit Niedrigwasser angesammelt wurde. Für die nächsten Hochwasser fallen diese Anreicherungen an Feinstoffmaterial im Flußbett aus und es wird sogar von einer Schwebstoffverarmung, die nach mehreren Hochwassern erreicht wird, gesprochen.

Tabelle 4: Tonmineralanteile der Schwebstoffe in Gew. \% (Fraktion $2 \mu$ ). Nr. 5, 6, 7, 8 und 9 sind Einzelproben, deren Schwebstoffgehalte abnehmend angeordnet sind. Nr. 10 sind Durchschnittsproben

\begin{tabular}{|c|c|c|c|c|c|c|c|}
\hline & & 10 & 10 & 24 & sp & - & \\
\hline Linth & Weesen Gäsi & 5 & 71 & 29 & - & - & - \\
\hline & & 6 & 73 & 27 & - & - & - \\
\hline & & 7 & 74 & 26 & - & - & 一 \\
\hline & & 8 & 72 & 28 & - & - & - \\
\hline & & 9 & 70 & 30 & - & - & - \\
\hline & & 10 & 72 & 28 & - & - & - \\
\hline Inn & Martinsbruck & 5 & 80 & 20 & - & - & - \\
\hline & & 6 & 75 & 25 & - & - & - \\
\hline & & 7 & 80 & 20 & - & - & - \\
\hline & & 8 & 76 & 24 & - & - & - \\
\hline & & 9 & 78 & 22 & - & - & - \\
\hline & & 10 & 73 & 27 & - & - & - \\
\hline Lütschine & Bönigen & 5 & 66 & 34 & - & - & - \\
\hline & & 6 & 70 & 30 & - & - & - \\
\hline & & 7 & 70 & 30 & - & - & - \\
\hline & & 8 & 75 & 25 & - & - & - \\
\hline & & 9 & 69 & 31 & - & - & - \\
\hline & & 10 & 65 & 35 & - & - & - \\
\hline Lonza & Blatten & 5 & 58 & 42 & - & - & - \\
\hline & & 6 & 60 & 40 & - & - & - \\
\hline & & 7 & 60 & 40 & - & - & - \\
\hline & & 8 & 62 & 38 & - & - & - \\
\hline & & 9 & 64 & 36 & - & - & - \\
\hline & & 10 & 62 & 38 & - & - & - \\
\hline Aare & Brienzwiler & 7 & 68 & 32 & - & - & - \\
\hline & & 8 & 73 & 27 & - & - & - \\
\hline & & 9 & 70 & 30 & - & - & - \\
\hline & & 10 & 72 & 28 & - & - & - \\
\hline Rhein & Bad Ragaz & 5 & 83 & 17 & - & - & - \\
\hline & & 6 & 85 & 15 & - & - & - \\
\hline & & 7 & 80 & 20 & - & - & - \\
\hline & & 8 & 86 & 14 & - & - & 一 \\
\hline & & 9 & 87 & 13 & - & - & - \\
\hline & & 10 & 79 & 21 & - & - & - \\
\hline Thur & Bütschwil & 5 & 60 & 13 & 15 & 12 & - \\
\hline & & 6 & 53 & 9 & 26 & 12 & - \\
\hline & & 7 & 62 & 11 & 13 & 14 & - \\
\hline & & 8 & 58 & 13 & 19 & 10 & - \\
\hline & & 9 & 67 & 22 & - & 11 & - \\
\hline & & 10 & 53 & 15 & 21 & 11 & - \\
\hline Arve & Genève & 10 & 76 & 24 & - & - & - \\
\hline Rhein & Schmitter & 10 & 79 & 21 & - & - & - \\
\hline $\begin{array}{c}\text { Grande } \\
\text { Eau }\end{array}$ & Aigle & 10 & 70 & 30 & - & - & - \\
\hline Borgne & La Luette & 10 & 63 & 37 & - & - & - \\
\hline Aare & $\begin{array}{l}\text { Interlaken } \\
\text { Unterseen }\end{array}$ & 10 & 71 & 29 & - & - & - \\
\hline Linth & Weesen Biäsche & 10 & 70 & 30 & - & - & - \\
\hline Aare & Stilli & 10 & 70 & 12 & - & 12 & 6 \\
\hline Rhône & Porte du Scex & 10 & 68 & 25 & - & 7 & - \\
\hline Rhône & Genève & 10 & 63 & 37 & - & - & - \\
\hline Aare & Brugg (AG) & 10 & 60 & 17 & - & 17 & 0 \\
\hline Aare & Brügg (BE) & 10 & 55 & 25 & 11 & 9 & - \\
\hline
\end{tabular}


Mineralzusammensetzung der Schwebstoffe einiger Schweizerflüsse

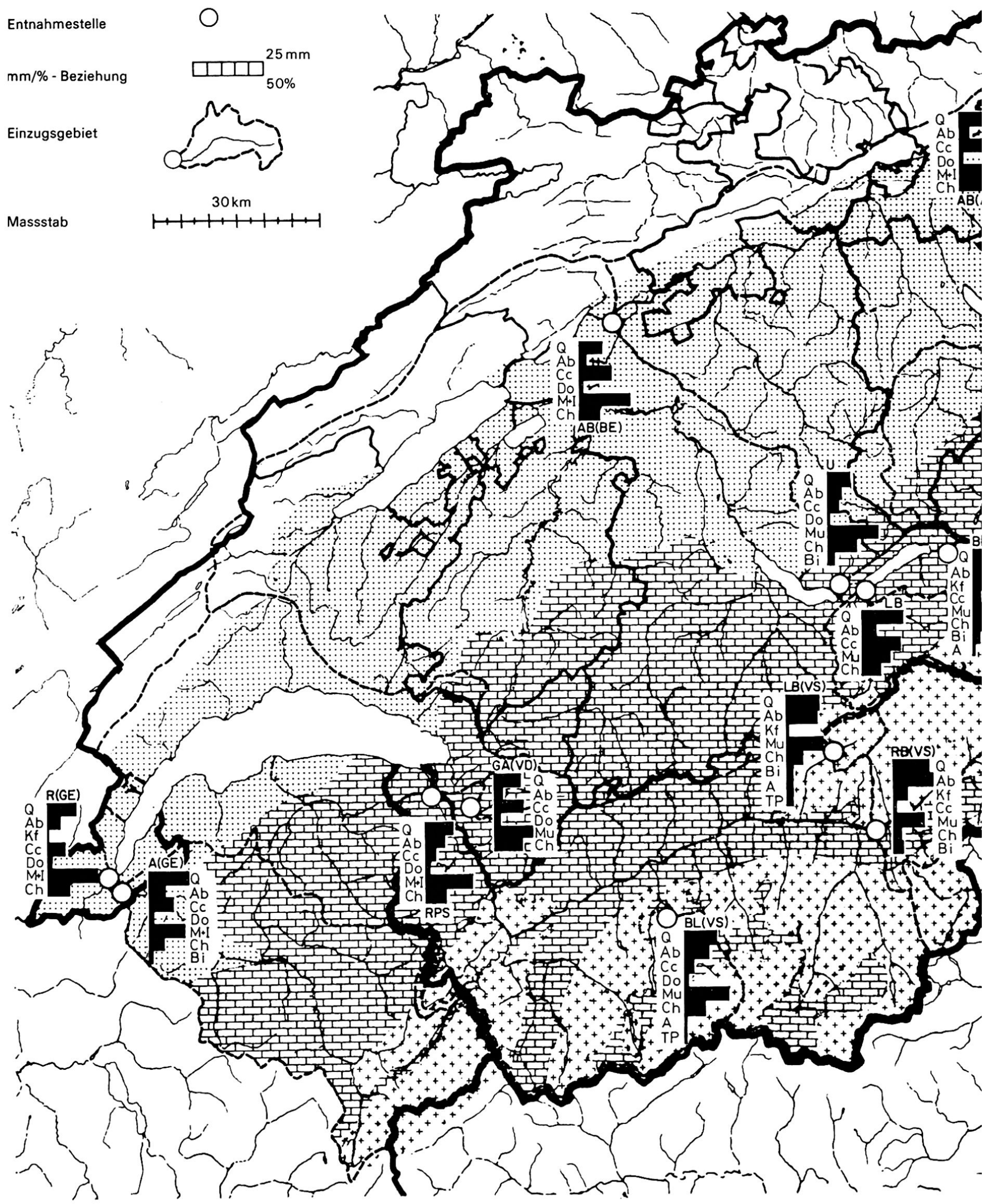




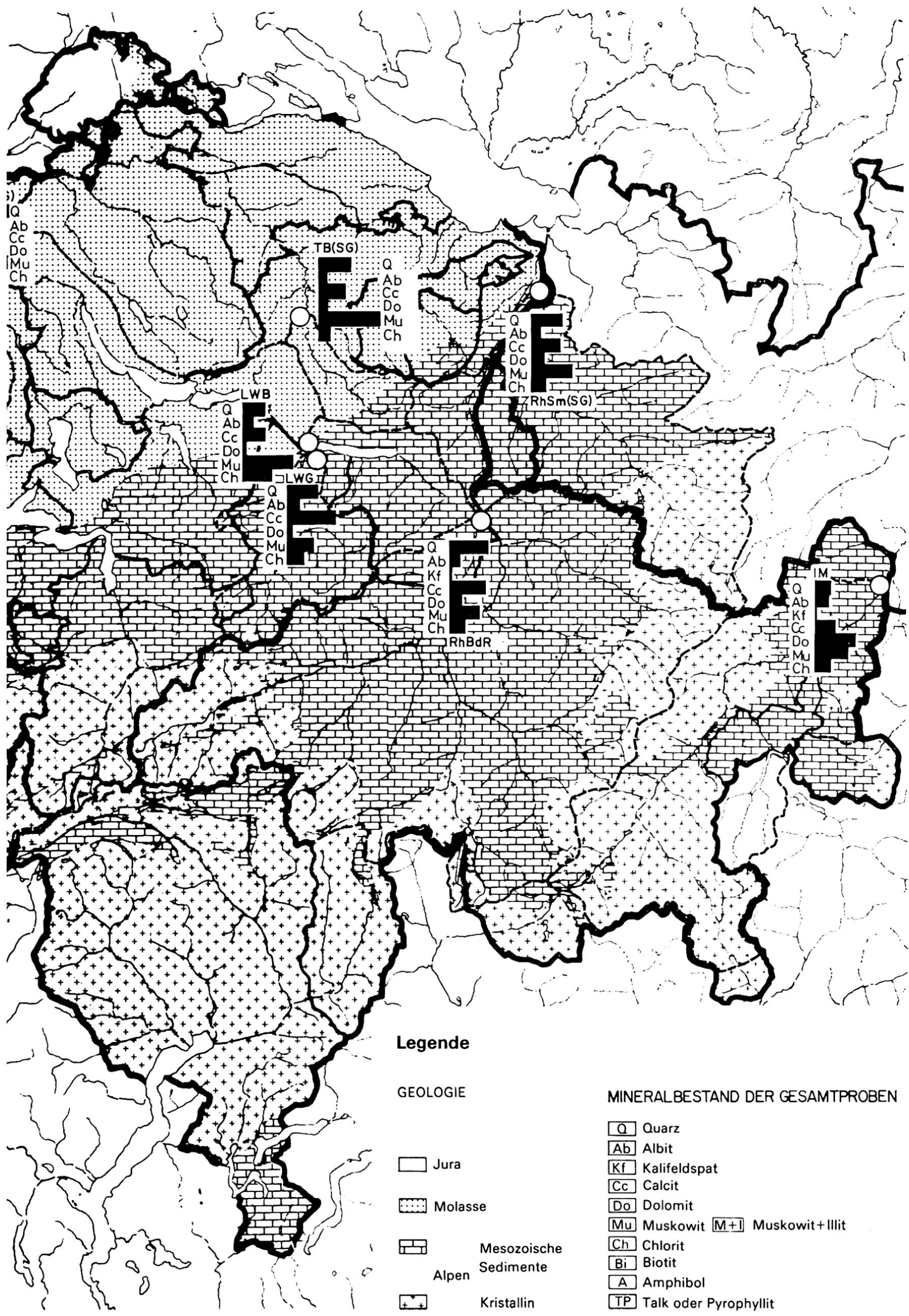


$\vec{A}$ Zusammensetzung der Tonfraktion von
Schwebstoffen einiger Schweizerflüsse

Entnahmestelle $\mathrm{O}$

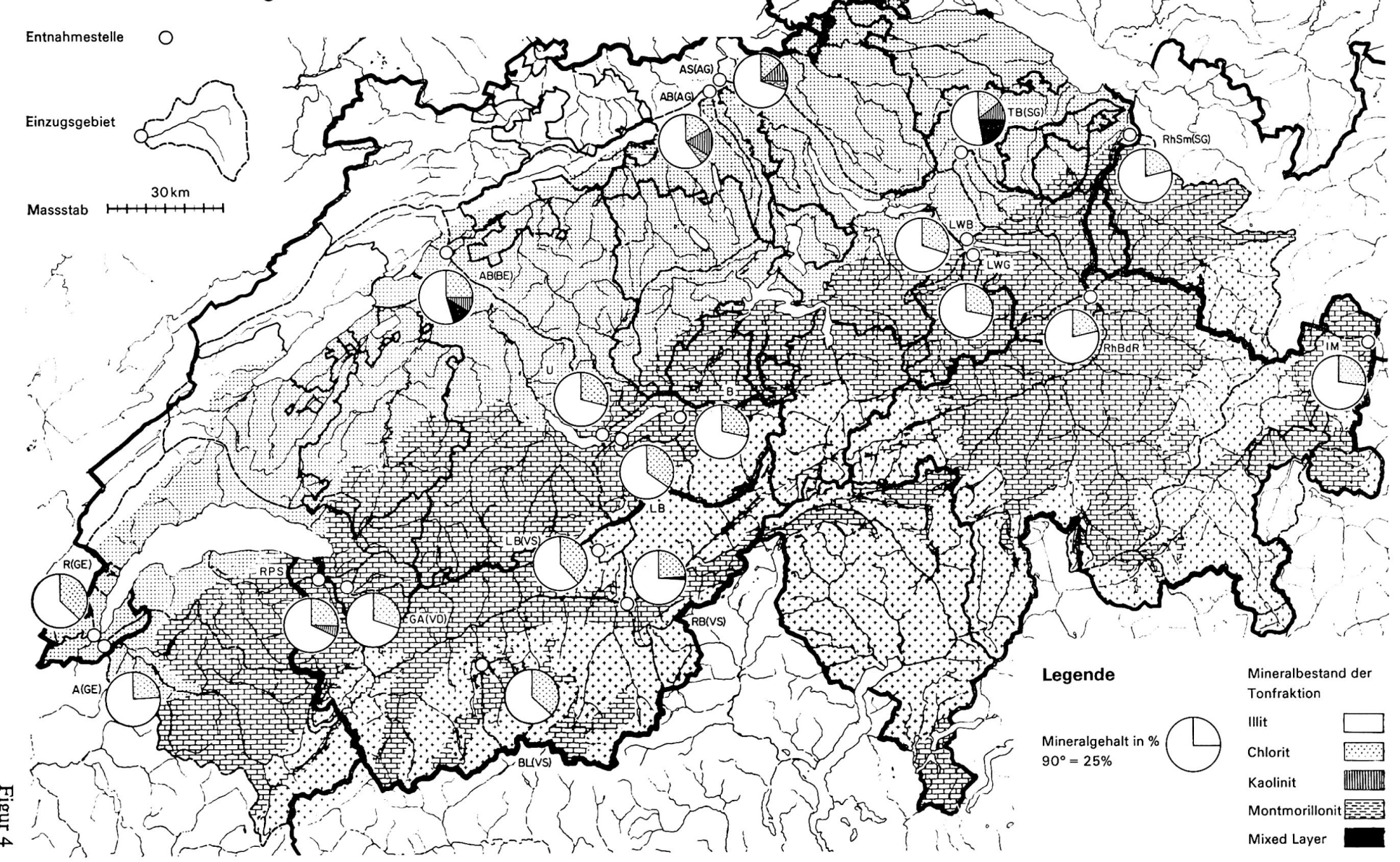




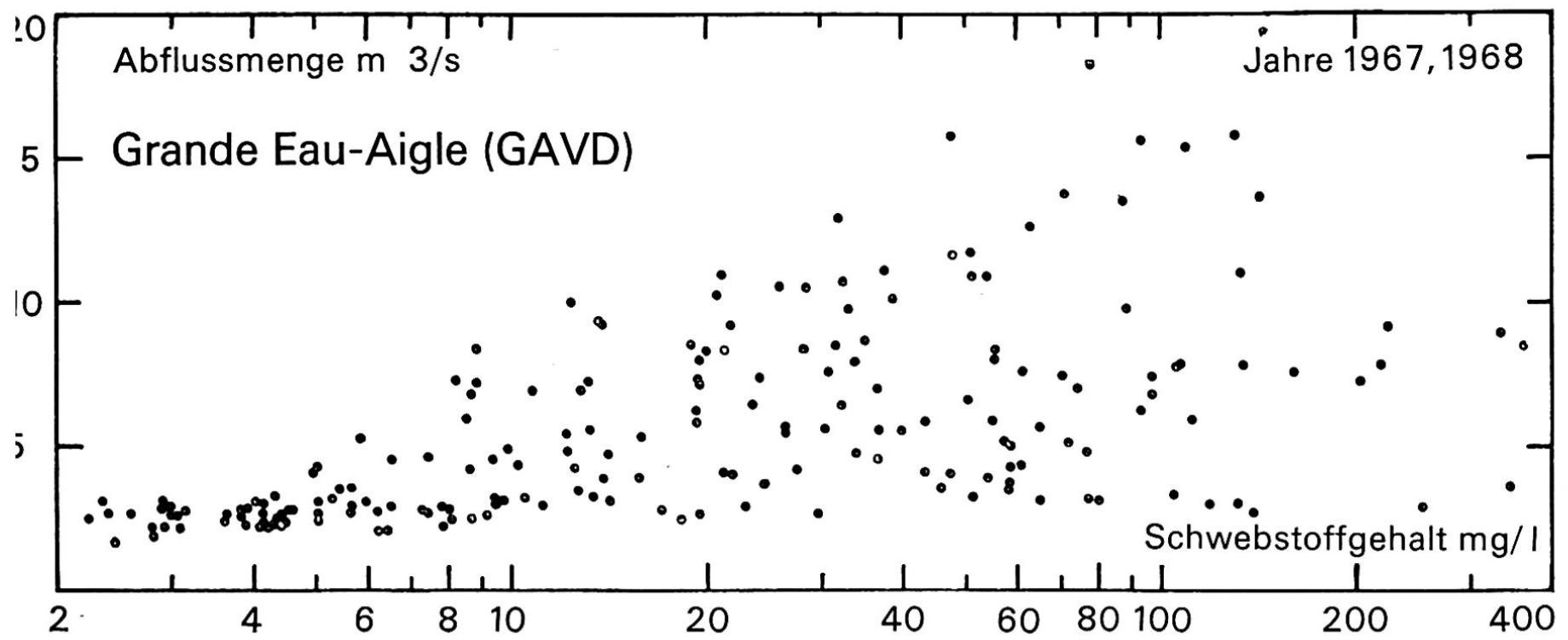

Figur 5

Die Faktoren a und b der oben erwähnten Gleichung sollen im folgenden erläutert werden.

Hohe a-Werte findet man in Gebieten mit starker Verwitterungsintensität. Müller und Förstner (1968) führen hier den der Literatur entnommenen Rio Puerco (Mexiko) mit einem a-Faktor von 80000 als typisches Beispiel auf. Dieser sei oft ausgetrocknet, führe aber bei Hochwassern extreme
Schwebstoffmengen mit sich. Unseren größten aWert weist die Entnahmestelle Rhône Porte du Scex mit 13,7 auf.

Die b-Werte zeigen die Erosionskraft der verschiedenen Flüsse.

Die Entnahmestelle Aare Brügg, welche kurz nach dem Ausfluß der Aare aus dem Bielersee lokalisiert ist, weist den geringsten unserer b-Werte, 0,36, auf.

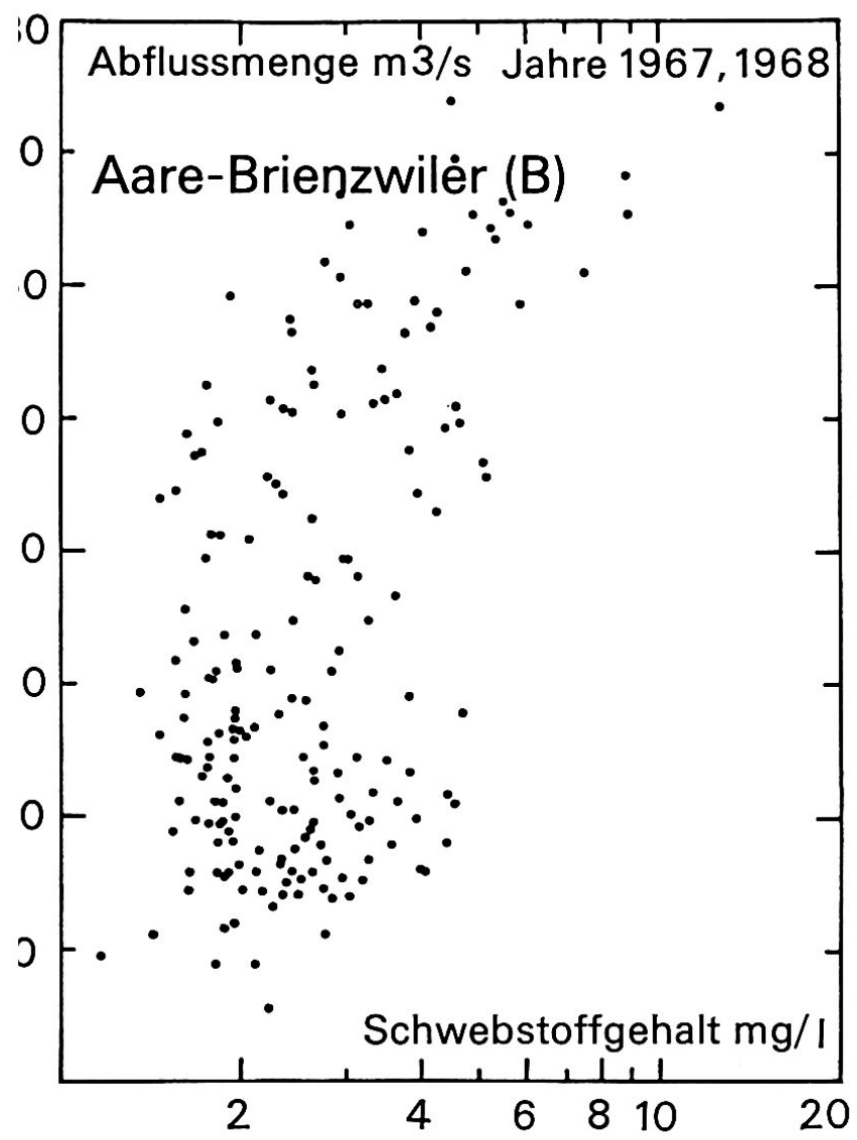

Figur 6

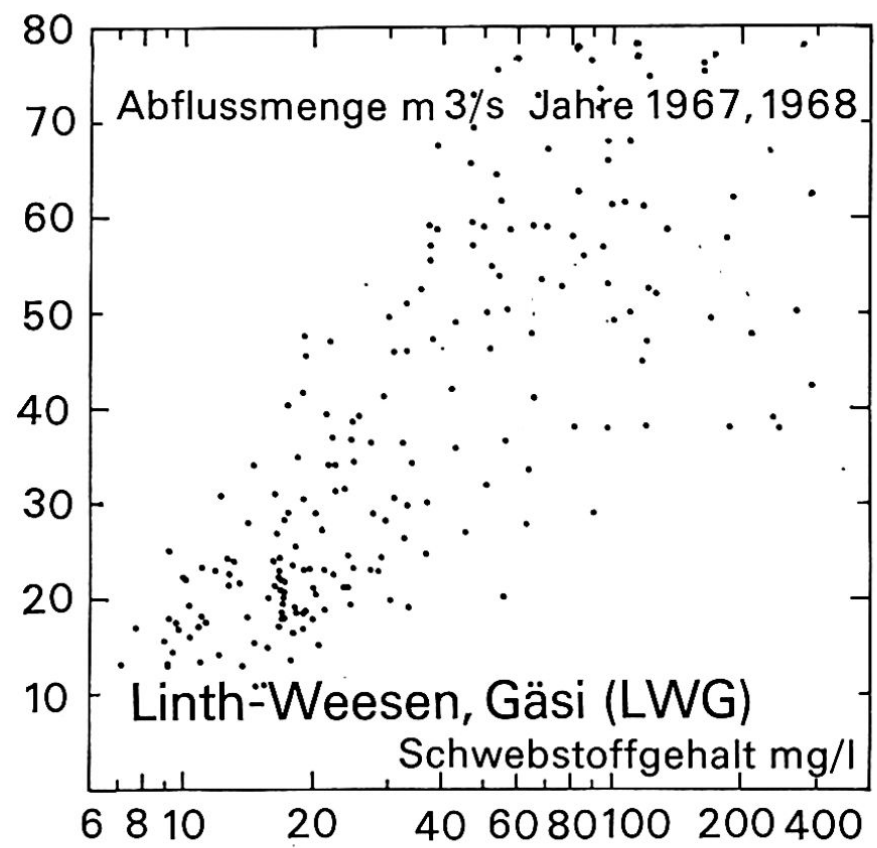

Figur 7 
Mittlere monatliche Schwebstoffbelastung einiger

Schweizerflüsse der Jahre 1964 - 1969

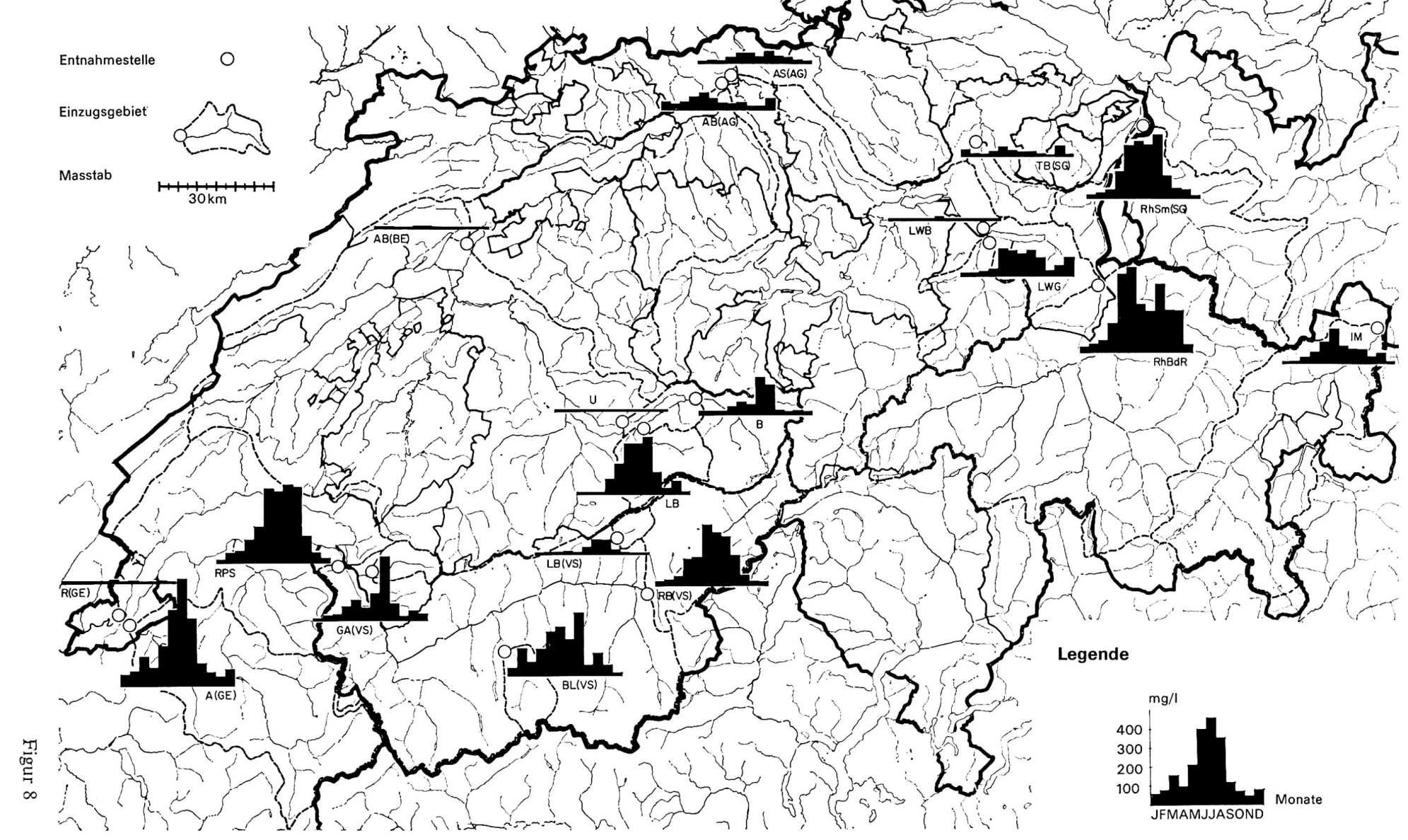


Er kommt dem b-Wert des Seerheins bei Konstanz, welchen Müller und Förstner (1968) in ihrer Arbeit aufführen, am nächsten. Die vom Gefälle und der Form des Flußbettes abhängige Erosionskraft ist hier praktisch nicht wirksam. Maximale b-Werte besitzen der Rhein bei Bad Ragaz mit 3,7 und bei Müller und Förstner (1968) der Alpenrhein bei Lustenau, Österreich mit 2,2. Diese Werte zeigen, wie stark der Rhein erodierend wirkt. Andere Stationen mit hohen b-Faktoren sind Borgne La Luette mit 2,5 und Arve Genève mit 2,4.

\section{Schwebstoffracht}

Die Schwebstoff- und Abflußmengen sowie deren Beziehungen zueinander sind für jeden Fluß mit den zu Beginn erwähnten Methoden relativ leicht bestimmbar. Anhand der gemessenen Daten kann die Schwebstofffracht und damit der Abtrag für die Alpen berechnet werden.

Im Eidg. Amt für Wasserwirtschaft ist eine Berechnungsmethode für die Schwebstofffracht entwikkelt worden (Eidg. Amt für Wasserwirtschaft, 1968). Bei dieser Methode ist der Schwebstoffwert $C$ für die nichtbeobachteten Tage mit einem Quotienten $\mathrm{K}$ interpoliert worden. Deutlich sind die bei der Berechnung getroffenen Annahmen hervorgehoben. Erstens sei für die Schwebstoffbelastungen angenommen worden, daß sie Mittelwerte im Flußquerprofil darstellen. Weiter sei mit einer annähernden Proportionalität zwischen Abflußmenge und Schwebstoffbelastung am Probenentnahmedatum gerechnet worden. Zusätzlich wird geraten, die $\mathrm{K}$-Werte den bei Hochwasser stark veränderten Abflußmengen anzupassen.

Wir haben in unseren Berechnungen für die Tage ohne Schwebstoffentnahme nicht mit dem Quotienten $\mathrm{K}$ interpoliert, sondern direkt die Schwebstoffmengen $\mathrm{C}^{\prime}$ in $\mathrm{gr} / \mathrm{m}^{3}$ mit den Tagesabflußmengen $\mathrm{Q}$ in $\mathrm{m}^{3} / \mathrm{s}$ und der Anzahl s/Tag multipliziert. Wir erhielten den Wert der Schwebstofftagesfracht (C'. Q gr/Tag), der für alle jene Tage, an denen man Schwebstoffproben entnommen hat, berechnet wurde. Es handelt sich durchschnittlich um 100

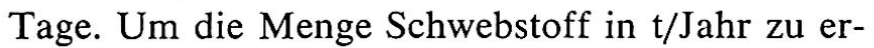
halten, haben wir mit einem Faktor $\left(\frac{365}{\approx 100 \cdot 10^{6}}\right)$ multipliziert. Unsere Werte für die Schwebstofffrachten sind alle etwas höher ausgefallen als diejenigen des Eidg. Amtes für Wasserwirtschaft.

Die Beträge der Schwebstofffrachten in $t / J a h r$ dienen zu weiteren langfristigen Berechnungen des Sedimenttransportes, welche von Bedeutung für die Abtragung der Alpen seit ihrer letzten Hebung Ende Miozän/Anfang Pliozän sind. In seiner Arbeit zeigt Jäckli (1958) anhand der Abtragungskur-

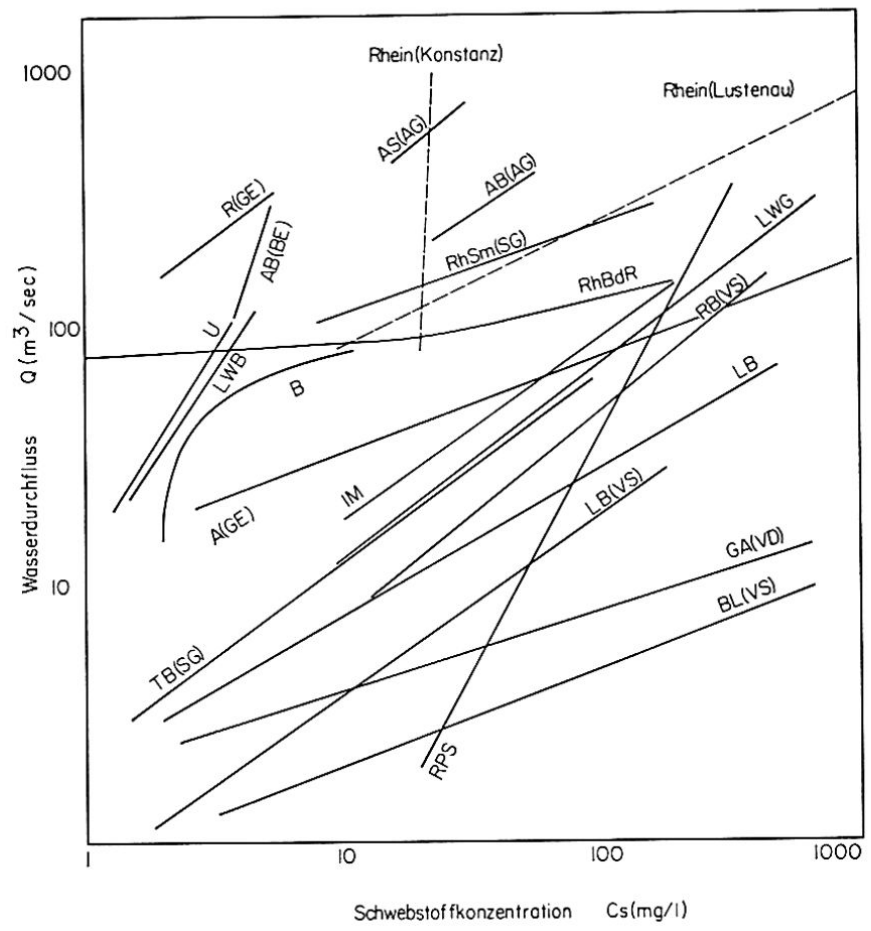

Figur 9. Beziehungen zwischen Schwebstoffkonzentration, Cs und Durchflußmenge $Q$. Cs $=\mathrm{a} \cdot \mathrm{Q}^{\mathrm{b}}$ Die schematischen Linien sind aus den Punktwolken der einzelnen Stationen ermittelt worden. Einige Punktwolken sind in den Fig. 5, 6 und 7 dargestellt. Die für die Linien verwendeten Abkürzungen sind in der Tabelle 1 erläutert.

ven einiger Alpenflüsse, wie in 3 bis 4 Mio. Jahren die mittlere Höhe der Einzugsgebiete auf die Hälfte reduziert worden ist.

\section{Abtragung}

In kristallinen Gebieten ist die Abtragung gering. In sedimentären Gebieten, in welchen z. B. Bündnerschiefer- oder Flyschvorkommen vorwiegen, wird viel Material abgetragen. Dieses liegt aufgelockert und wenig erosionsresistent für den Mittransport bereit.

Ein ausschließlich kristallines Einzugsgebiet, mit einer geringen mittleren Schwebstoffbelastung, hat nur die Lonza Blatten, Fig. 8. Der Abtragungsfaktor, berechnet aus der Schwebstofffracht ( $\mathrm{t} / \mathrm{Jahr}$ ), der Fläche des Einzugsgebietes $\left(\mathrm{km}^{2}\right)$ und einer mittleren Dichte von $2,5\left(\mathrm{gr} / \mathrm{cm}^{3}\right)$, ist mit $0,03 \mathrm{~mm} /$ Jahr einer der niedrigsten Werte in Tabelle 5. Alle andern Einzugsgebiete erstrecken sich über zwei oder drei geologische Hauptregionen (Diss. Peters 1971). Sie weisen daher unterschiedliche, vielfältige Erosionsbedingungen innerhalb der einzelnen Gebiete auf, welche im folgenden besprochen werden sollen. Neben den Gebieten mit geringster Ero- 
sion ließen sich alle Abstufungen an Erosionsintensitäten feststellen. Hier aber teilen wir nur ein in Gebiete mit mittlerer und starker Erosion und Gebiete, in denen der Fluß nicht erodiert, sondern akkumuliert.

Gebiete mit mittlerer Erosionswirkung sind Schuttlieferanten von stets kleineren Mengen oder gelegentlich, bei Hochwasser, extrem hohen Mengen. Es handelt sich hier um bewachsene Schuttpartien an Hängen über dem Flußbett oder im Flußbett selber. Eine starke Erosionswirkung wird in den obenerwähnten Schiefergebieten und auf unbewachsenen Schuttflächen verzeichnet. Zu den Akkumulationsgebieten, wo fluviatile Aufschüttungen überwiegen, gehören Talsohlen und Becken, Seitentälerschuttkegel, künstliche Verbauungen und schließlich die Ablagerungsräume der großen Seen. In der Fig. 8 fallen die vier nach Seeausfluß situierten Entnahmestellen Linth Weesen Biäsche, Aare Brügg, Interlaken/Unterseen und Rhône Genève, bei denen die Säulendarstellungen der mittleren Schwebstoffbelastung praktisch zu horizontalen Linien werden, sofort auf. Der Abtragungsfaktor wurde für diese vier Einzugsgebiete dementsprechend gar nicht berechnet (Tabelle 5). In der vorliegenden Arbeit konnte nicht auf die einzelnen Seen der Einzugsgebiete unserer Flüsse eingegangen werden. Jäckli (1957) zählte allein im bündne- rischen Rheingebiet 350 Seen, von denen die Einzugsgebiete der 37 größten 2,2\% der Gesamtfläche ausmachen. Er stellt fest, daß seit dem letzten Gletscherrückzug viele Seen verlandet sind und heute vorwiegend Seen im Kristallin vorkommen, mit $\mathrm{Zu}$ flüssen von unterdurchschnittlicher Schuttführung. Jäckli hat die innerhalb des Einzugsgebietes abgelagerten, rezenten Sedimente geschätzt und sie zu dem zugehörigen Abtragungsbetrag der Deltasedimente des Rheins addiert. Er erhielt einen Wert von $0,9 \mathrm{~mm} / \mathrm{Jahr}$, um den das Erosionsgebiet des Bündnerrheins erniedrigt wird. In unseren Untersuchungen haben wir die in den Einzugsgebieten abgelagerte Sedimentation nicht quantitativ berücksichtigt. Unsere Abtragungsintensitäten bewegen sich zwischen 0,01 und $0,51 \mathrm{~mm} / \mathrm{Jahr}$ (Tabelle 5). Der maximale Wert von $0,51 \mathrm{~mm} / \mathrm{Jahr}$ ist aber nicht repräsentativ, weil im Einzugsgebiet Rhein Bad Ragaz im Jahre 1965 außerordentlich viel Niederschläge fielen. Als Folge davon sind extrem hohe Abflußmengen verzeichnet worden, welche zum maximalen Wert geführt haben. Im Vergleich dazu ist die Abtragungsintensität von Rhein Schmitter im Jahre 1968 von $0,13 \mathrm{~mm} / \mathrm{Jahr}$ eher gerechtfertigt. $\mathrm{Zu}$ dem Abtrag, welcher den transportierten Schwebstoffen entspricht, ist noch das in den Flüssen als chemische Lösung mitgeführte Material zu addieren. In den Hydrographischen Jahrbüchern,

Tabelle 5: Schwebstoffjahresfracht und Abtragung

Entnahmestelle

Jahr

$\begin{array}{ll}\text { Rhône } & \text { Brigerbad } \\ \text { Linth } & \text { Weesen Gäsi } \\ \text { Inn } & \text { Martinsbruck } \\ \text { Lütschine } & \text { Bönigen } \\ \text { Lonza } & \text { Blatten } \\ \text { Aare } & \text { Brienzwiler } \\ \text { Rhein } & \text { Bad Ragaz } \\ \text { Thur } & \text { Bütschwil } \\ \text { Arve } & \text { Genève } \\ \text { Rhein } & \text { Schmitter } \\ \text { Grande Eau } & \text { Aigle } \\ \text { Borgne } & \text { La Luette } \\ \text { Aare } & \text { Interlaken } \\ \text { Linth } & \text { Unterseen } \\ \text { Aare } & \text { Weesen Biäsche } \\ \text { Rhône } & \text { Stilli } \\ \text { Rhône } & \text { Porte du Scex } \\ \text { Aare } & \text { Genève } \\ \text { Aare } & \text { Brugg (AG) } \\ \end{array}$

1965

1965

1965

1965

1968

1965

1965

1965

1965

1968

1968

1968

1965

1965

1965

1965

1965

1965

1965
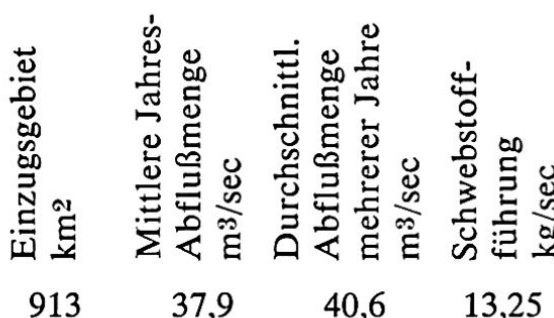

616

1945

379

78

554

4455

303

1983

6119

132

231

1140

1061

17625

5220

7987

11750

8317

65

696,0

161,0

245,0

398,0

303,0

\section{6,3}

551,0

180,0

239,0

306,0

243,0
35,62

73,50

26,9

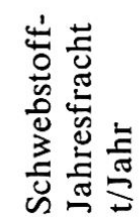

$\begin{array}{rrr}37,9 & 40,6 & 13,25 \\ 37,4 & 33,2 & 8,12 \\ 59,7 & 57,8 & 10,90 \\ 20,3 & 18,8 & 7,60 \\ 4,0 & 4,5 & 0,18 \\ 35,5 & 33,4 & 12,57 \\ 177,0 & 145,0 & 179,50 \\ 18,8 & 13,8 & 2,38 \\ 85,7 & 79,3 & 39,10 \\ 256,0 & 238,0 & 63,70 \\ 5,9 & 5,2 & 0,75 \\ 3,1 & 7,3 & 0,14 \\ 66,9 & 61,4 & \end{array}$

374000

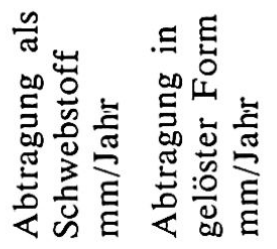

255000

0,16

0,17

0,07

$\begin{array}{ll}344000 & 0,07 \\ 233400 & 0,25\end{array}$

$5660 \quad 0,03$

$397000 \quad 0,29$

$5640000 \quad 0,51$

$74900 \quad 0,10$

$1078000 \quad 0,22$

$2035000 \quad 0,13$

$23500 \quad 0,07$

$4420 \quad 0,01$

0,06

0,08

1116000

0,03

0,07

2320000

0,18

0,07

830000

0,03 
Eidg. Amt für Wasserwirtschaft, sind die Werte der Gesamthärte des Wassers von 5 unserer Entnahmestellen angegeben. Die gelösten Karbonate, besonders $\mathrm{CaCO}_{3}$ und etwas $\mathrm{MgCO}_{3}$, sind vorherrschend, während nur wenig Silikate gelöst sind. Die durch diese gelösten Stoffe bedingte Abtragung beträgt 0,06-0,08 mm/Jahr und muß zu der Abtragungsintensität - berechnet aus den Schwebstoffen - hinzugezählt werden. Jäckli (1958) erhielt als chemische Abtragung für das gesamte bündnerische Rheingebiet einen Wert von $0,052 \mathrm{~mm} / \mathrm{Jahr}$ und Uetrecht (1906) für das Einzugsgebiet der Rhône einen solchen von $0,066 \mathrm{~mm} / \mathrm{Jahr}$.

Die niedrigen Werte für gelöste Silikate und die hohen Karbonatanteile zeigen, daß die chemische Abtragung, entsprechend derjenigen von Schwebstoffen, in sedimentären Gebieten eine große Rolle spielt und in kristallinen Gegenden kaum ins Gewicht fällt.

\section{Literaturangaben}

Berry, R. W., Brophy, G. P. u. Naqash, A. (1970): Mineralogy of the Suspended Sediment in theTigris, Euphrates, and Shatt-al-Arab Rivers of Iraq, and the Recent History of the Mesopotamian Plain. Journ. of Sed. Petr. Vol. 40, Nr. 1.

Eidg. Amt für Wasserwirtschaft: Hydrographische Jahrbücher der Jahre 1964-1968.

Eidg. Amt für Wasserwirtschaft (Unterabteilung Landeshydrographie) (1967): Anleitung für die Feldarbeiten bei der Wassermessung mit hydrometrischen Flügeln (ohne Mehrflügelmessung).

Eidg. Amt für Wasserwirtschaft (Unterabteilung Landeshydrographie) (1968): Studie: «Úber die Beziehung zwischen Abfluß und Schwebstofführung und über eine angenäherte Berechnung von Schwebstofffrachten».

Eidg. Amt für Wasserwirtschaft: Report Nr. 7 (1943): A Study of New Methods of Suspended Sediment Samples. Hydraulic Laboratory, University of Iowa, Iowa City.

- Report Nr. 14 (1963): Determination of Fluvial Sediment Discharge. St. Anthony Falls Hydraulic Laboratory, Minneapolis, Minnesota.

Beide sind beim Superintendent of Documents, U.S. Government Printing Office Washington D.C., 20402, nicht mehr erhältlich!

Füchtbauer, H. (1964): Sedimentpetrographische Untersuchungen in der älteren Molasse nördlich der Alpen. Eclogae geol. Helv. 57/1.

Jäckli, H. (1957): Gegenwartsgeologie des bündnerischen Rheingebietes. Beitr. zur. Geol. d. Schweiz. Geotechn. Serie. 36. Lieferung. Kommissionsverlag Kümmerly \& Frey, Bern.

Jäckli, H. (1958): Der rezente Abtrag der Alpen im
Spiegel der Vorlandsedimentation. Eclogae geol. Helv., Vol. 51, 2.

Johns, W. D., Grim, R. E. und Bradley, W. F. (1954): Quantitative estimations of clay minerals by diffraction methods. Journ. of Sed. Petr. Vol. 24, Nr. 4.

Klug, H. P. und Alexander, L. E. (1954): X-Ray Diffraction Procedures for Polycrystalline and Amorphous Materials. N.Y. John Wiley \& Sons, Inc.

Kubler, B. (1967): La cristallinité de l'Illite et les zones tout à fait supérieures du métamorphisme. Etages tectoniques, Colloque Neuchâtel, p. 105122.

Müller, G. und Förstner, U. (1968): Sedimenttransport im Mündungsgebiet des Alpenrheins. Geol. Rundschau. Band 58; 1. Verlag Ferdinand Enke. Nydegger, P. (1967): Untersuchungen über Feinstofftransport in Flüssen und Seen, über Entstehung von Trübungshorizonten und zuflußbedingten Strömungen im Brienzersee und einigen Vergleichsseen. Beitr. zur Geol. der Schweiz. - Hydrologie - Nr. 16.

Peters, B. (1971): Untersuchungen über Zusammensetzung und Transport von Schwebstoffen in einigen Schweizer Flüssen. Diss. phil. nat. Bern.

Peters, Tj. (1969): Terminologie einiger Glazialablagerungen im schweizerischen Mittelland. Eclogae geol. Helv. Vol. 62; 2.

Rantz, S. E. und Moore, A. M. (1965): Floods of December 1964 in the far western states. U.S. Geol. Survey Water Resources Division Open-file Report. Menlo Park, California.

Uetrecht, E. (1906): Die Ablation der Rhone in ihrem Walliser Einzugsgebiete im Jahre 1904/05. Diss. Univ. Bern.

Vernet, J.-P. (1959): Etudes sédimentologiques et pétrographiques des Formations Tertiaires et Quaternaires de la partie occidentale du Plateau suisse. Eclogae geol. Helv. 1958, Vol. 51; 3.

Wenk, E. (1962): Plagioklas als Indexmineral in den Zentralalpen. Die Paragenese Calcit - Plagioklas. Schweiz. Mineralog. Petrogr. Mitt. Band 42; 1 .

Whetten, J. T., Kelley, J. C. und Hanson, L. G. (1969): Characteristics of Columbia River Sediment and Sediment Transport. Journ. of Sed. Petr. Vol. 39; 3.

de Wolff, P.-M. (1951): Contributions to the Theory and Practice of Quantitative Determinations by the X-ray Powder Diffraction Method. Diss. Technical University of Delft.

Adresse der Verfasserin:

Frau B. Peters-Kümmerly

Falkenweg 9, 3012 Bern 